\title{
Molecular regulation of miRNAs and potential biomarkers in the progression of hepatic steatosis to NASH
}

\begin{abstract}
Increasing evidence suggests that microRNAs regulate diverse biological functions in the liver and play a very important function in metabolic-related disorders such as nonalcoholic fatty liver disease via regulating their target genes expression. In this review, we summarized the most recent progress in identification of miRNAs involving in the progression of liver steatosis and discussed the possible mechanisms by which miRNAs contribute to the diverse pathogenic liver injuries. We provide insights into the functional network of miRNAs by connecting miRNAs, their targets and biological pathways associated to hepatic steatosis and fibrosis, with important implications for our understanding of phenotypic-based disease pathogenesis. We also discuss the possible roles and challenges of miRNAs as biomarkers for drug-induced liver injury.
\end{abstract}

Keywords: biomarker $\bullet$ drug-induced liver disease $\bullet$ fibrosis $\bullet$ miRNA $\bullet$ nonalcoholic fatty liver disease $\bullet$ steatohepatitis $\bullet$ steatosis

Nonalcoholic fatty liver disease (NAFLD) is a clinicopathological change characterized by the accumulation of triglycerides in hepatocytes and is increasing with obesity, Type 2 diabetes mellitus, hyperlipidemia and insulin resistance [1]. The pathogenesis of NAFLD has often been interpreted by the 'double-hit' hypothesis. The primary insult or the "first hit" includes lipid accumulation in the liver, while during the second hit, pro-inflammatory mediators induce inflammation, hepatocellular injury and fibrosis. Several factors have been recently described as playing a key role in NAFLD pathogenesis [2], however, the molecular mechanism underlying NAFLD progression is not completely understood.

MicroRNAs (miRNA) are small noncoding RNAs of approximately 22 nucleotides to regulate post-transcriptional gene activities by controlling the translation of mRNA into proteins [3]. There are more than 2500 miRNAs reported in the human genome and thousands of their target mRNAs, highlighting the important regulatory roles of miRNAs in cell development, differentiation, proliferation and apopto- sis [4,5]. Most miRNAs are evolutionarily conserved in closely related species and some even show conservation between invertebrates and vertebrates [3]. Because of short sequences, multiple miRNAs may repress the expression of a specific gene simultaneously by targeting different sequence regions; likewise, a single miRNA may be able to regulate the expression of dozens or even hundreds of targets at the same time [6].

The biological importance of miRNAs, initially demonstrated in cancer [7], was continually discovered in many other pathologies such as nervous system disorder [8], cardiac diseases [9], viral infections [10], diabetes [11] and liver diseases including drug-induced liver injury (DILI) [12,13]. In addition, it has been reported that the tissue-specificity of a miRNA is correlated with the number of diseases $[14,15]$. For instance, a cluster of miRNAs-17-92 is typically expressed in human B-cell lymphomas [16]. Moreover, overexpression of miR-195 during cardiac hypertrophy results in pathological cardiac growth and heart failure, while miR-199a is expressed in cardiomyocytes where it maintains cell size
Yuping Wang ${ }^{* 1}$, Zhichao Liu', Wen Zou', Huixiao Hong', Hong Fang ${ }^{2} \&$ Weida Tong **,1 'Division of Bioinformatics \& Biostatistics, National Center for Toxicological Research, US FDA, 3900 NCTR Road, Jefferson, AR 72079, USA

2Office of Scientific Coordination, National Center for Toxicological Research, US FDA, 3900 NCTR Road, Jefferson, AR 72079, USA *Author for correspondence: Tel.: +1 8705437340 Yuping.wang@fda.hhs.gov **Author for correspondence: Tel.: +1 8705437142 Weida.tong@fda.hhs.gov 
and plays a role in the regulation of cardiac hypertrophy [17]. Such miRNA expression signatures may be able to differentiate human malignancies according to their developmental origin, which has important clinical implications. These findings can not only help understand the association between miRNAs and human diseases, but also suggest miRNAs as potential biomarkers for diagnosis and prognosis of diseases.

Currently, the definitive diagnosis of NAFLD is based on histologic examination of liver biopsy samples. However, ethical considerations as well as inherent risks associated with this procedure limit its widespread applicability as a screening test in apparently healthy individuals or even for those with significant risk factors. miRNAs were reported to be able to regulate adipocyte differentiation [18], lipid metabolism [19,20] and glucose resistance [21] to exert influence on metabolic pathways that were also considered to be involved in the pathogenesis of NAFLD. Therefore, it is plausible that certain miRNAs participate in the pathogenesis of NAFLD. Several studies identified specific miRNA expression profiles associated with different histological features of NAFLD, both in animal models and in patients [22,23]. Therefore, specific assortments of certain miRNAs could have enormous diagnostic potentiality. Facilitated by high-throughput genomics and bioinformatics in conjunction with traditional molecular biology techniques and animal models, miRNA research is now positioned to make the transition from laboratories to clinical setting to deliver profound benefits to the public health [24]. In this study, we focus on the association between miRNAs and the different molecular process involved in NAFLD. We summarized and discussed the regulatory function of miRNAs through interaction with their target genes and nuclear receptors associated to the progress of NAFLD, from hepatic steatosis, steatohepatitis and fibrosis. The information gathered here will facilitate further researches to complete gaps between the pathogenesis and biomarkers for diagnosis and treatment emerging from advent technologies. With this new paradigm, new biomarkers that are able to discriminate the progression of hepatic steatosis into fibrosis and cirrhosis will guide further studies of the roles of miRNAs in the NAFLD pathogenesis and the disease-specific miRNAs might become potential targets for therapeutic intervention.

\section{General progress of NAFLD}

NAFLD is a clinicopathological term with a spectrum of abnormalities ranging from simple hepatic steatosis to nonalcoholic steatohepatitis (NASH), fibrosis and cirrhosis [25]. The disease starts with accumulation of fatty acid in the hepatocytes which weakens the adaptive phase hepatocytes and makes them more susceptible to attacks followed by lipid peroxidation secondary to oxidative stress. Inflammation is ultimately responsible for the cellular damage, leading to liver fibrosis and cirrhosis, even hepatocarcinoma. One-third of patients with NAFLD develop NASH, of which about 9 to $20 \%$ progress to cirrhosis over a period of 5-10 years [26]. Although exact pathogenesis and underlying mechanism of steatosis are not fully understood, multiple factors including genetics and epigenetics play a role in the progression of NAFLD [27]. Growing evidence demonstrated that miRNAs may involve in each step from steatosis to fibrosis as indicated at Figure 1.

The emerging role of miRNAs in hepatic metabolic functions has led several studies to investigate the role of miRNAs in NAFLD pathogenesis. Regulatory miRNAs demonstrated their function to mediate or amplify the pathogenic processes that lead to hepatic steatosis, fibrosis or cirrhosis [22,28-30]. In addition, it is thought that miRNAs are crucial for regulating gene expression during metabolic-related disorders, including NAFLD. These studies bring insightful views into this complex disorder and have led to the development of novel therapeutic and diagnostic strategies that might enable a personalized approach in the management of this disease.

\section{Regulatory function of miRNAs on their target genes in the pathogenesis of hepatic steatosis}

miRNAs are important post-transcriptional regulators in different pathophysiological processes of liver steatosis [27]. Typically, they repress target gene expression through effect on the mRNA stability or translation. To date, there is growing evidence and experimental studies which have specifically explored the involvement of miRNAs in the mechanism associated with NAFLD development by regulating corresponding genes [20,31]. For example, the results from our ongoing studies on potential gene targets using the target prediction algorithms and the pathway analysis tools have revealed that around 54 miRNAs regulate 107 genes involved in the pathogenesis of hepatic steatosis (unpublished data). In addition, a panel of miRNAs has been experimentally validated to regulate genes via various mechanisms including interruption of fatty acid oxidation, lipogenesis, signaling events, lipid transport, lipid drop protein (Supplementary Table 1). For instance, miR-122 is a liver specific miRNA correlating hepatic fatty acid oxidation and cholesterol synthesis rate. Here, we summarized the most recent findings on the regulatory functions of miRNAs in NAFLD at Table 1. Notably, a recent study explored 


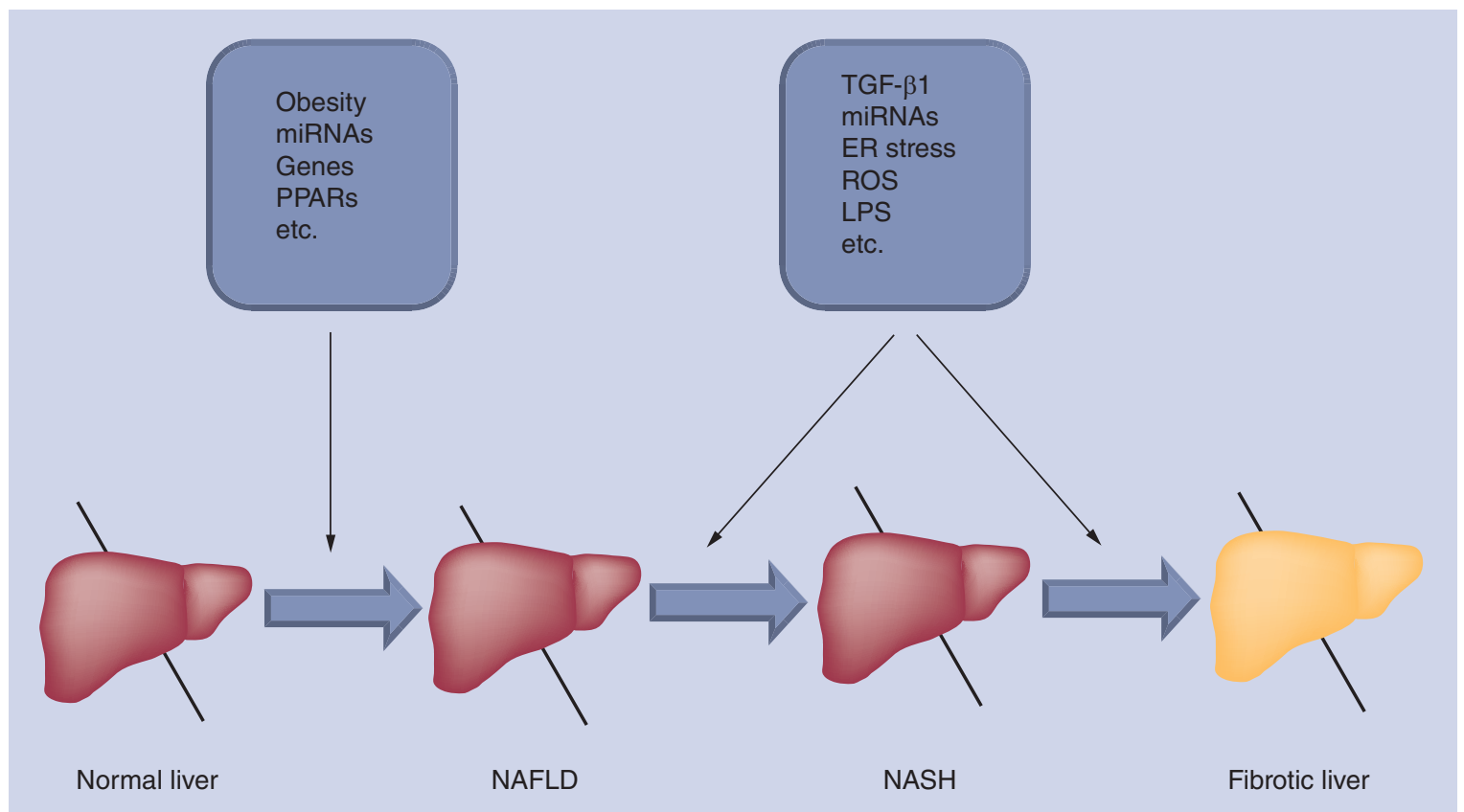

Figure 1. Schematic illustration of nonalcoholic fatty liver disease/nonalcoholic steatohepatitis progression.

Beginning with the simple hepatic steatosis, over time may progress to nonalcoholic steatohepatitis (NASH) and fibrosis. miRNAs involved in each step of the progression through interaction with their target gene or biological molecules, such as TGF- $\beta 1$.

the new function of miR-33, including miR-33a and miR-33b over lipid metabolism and fatty acid oxidation through regulation of sterol regulatory elementbinding protein. miR-33 is a family of miRNA precursors, which are processed by the Dicer enzyme to give mature miRNAs and have recently been shown to regulate lipid homeostasis in concert with their host genes [32,33]. The sequence of miR-33 is identical, and the stem-loop of the pre-miRNA is highly conserved in mammals and regulate liver steatosis through its target of Sterol regulatory element-binding transcription factor (SREBP) $[34,35]$. SREBP are the predominant transcription factors controlling the synthesis of cholesterol and fatty acids in the liver through controlling three risk factors of metabolic syndrome, namely levels of high-density lipoprotein (HDL), triglycerides (TG) and insulin signaling [36]. The discovery of coordinated regulation of cholesterol levels by miR-33a and the SREBP2 host gene product has prompted investigation into whether the SREBP host genes and their intronic miRNAs might act together more broadly to control not only cholesterol but also fatty acid and lipid homeostasis in an integrated manner $[35,37,38]$. Taken together, these results reveal an extensive and integrated network of functional interactions between the SREBP transcription factors and their intronic miRNAs (miR-33a and miR-33b) to regulate cholesterol and lipid homeostasis. Figure 2 schematically illustrated the regulation process of miR-33 and target genes on lipid metabolism.

\section{Cross-talking between miRNAs and PPARs in progress of NAFLD}

The key element in the process of lipogenesis and lipolysis in adipose and nonadipose tissues is mediated by peroxisome-proliferator-activated receptors (PPARs). PPARs are ligand-dependent transcription factors and have been shown to have critical roles in fatty acid oxidation, triglyceride synthesis and lipid metabolism $[39,40]$ - making them important in liver steatosis and DILI. Three major isoforms of PPARs (PPAR- $\alpha$, PPAR $-\beta / \delta$ and PPAR- $\gamma$ ) have been characterized to date. The transcriptional activity of the various PPARs isoforms in physiological and pathological situations is regulated by other numerous and complex epigenetic, transcriptional and post-transcriptional mechanisms [41,42]. Dysregulations of the expression, or activity, of specific PPAR isoforms in the liver are believed to be critical to the development metabolic disorders [43]. Particularly, PPAR $\alpha$ is abundantly expressed in hepatocytes as a transcriptional regulator of genes and involves in peroxisomal and mitochondrial $\beta$-oxidation, fatty acid (FA) transport and hepatic glucose production. PPAR- $\beta$ is markedly expressed in the brain, adipose tissue and skin [44]. In contrast to PPAR- $\alpha$, PPAR- $\gamma$ is present in adipose tissue and becomes elevated in fatty livers. PPAR- $\gamma$ is expressed in adipose tissue and not abundantly expressed in liver under normal conditions, but generally increased in steatotic livers of both animal models of obesity and human obese patients [45]. The epigenetic regulation of PPARs expression and activity 
Table 1. Most recent founding on the functions of miRNAs in steatosis.

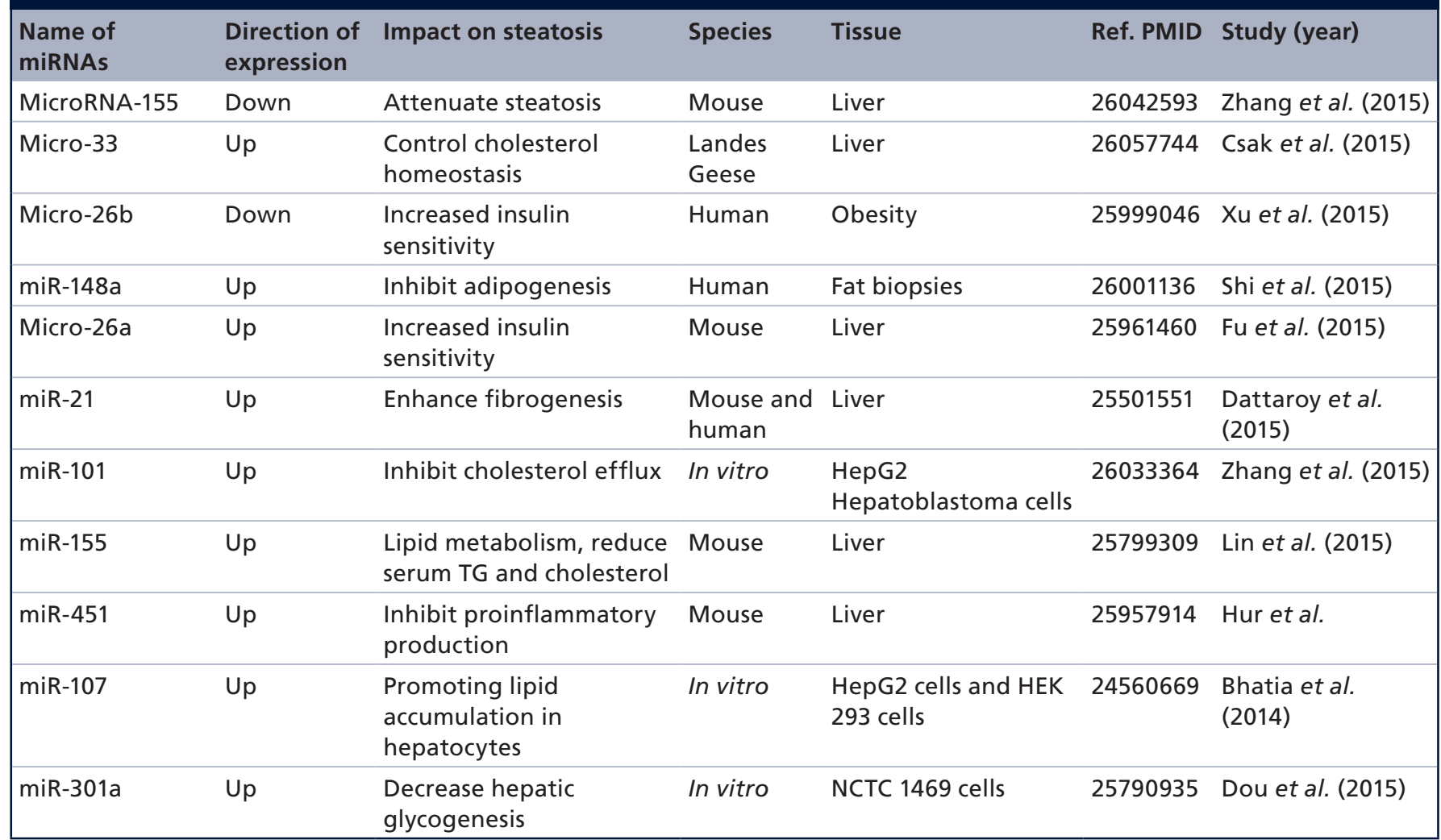

by miRNAs is a new field of research [42]. Accumulating evidence now suggests that alterations of the expression/activity of PPARs isoforms by distinct miRNAs could represent critical molecular mechanisms involved in the physiopathology of each organ undergoing a PPARs-dependent control [46,47]. Many PPAR- $\alpha$ target genes are involved in fatty acid metabolism in tissues such as brain, heart and liver with high oxidative rates $[48,49]$. PPAR $\alpha$ activation, in combination with PPAR- $\beta / \delta$ agonism, improves steatosis, inflammation and fibrosis in preclinical models of NAFLD [50], considering to be a new potential therapeutic area.

Recently, interaction of miRNAs and PPAR $\gamma$ has been extensively studied and the results demonstrated that liver PPAR $\gamma$ regulates triglyceride homeostasis, contributing to hepatic steatosis, but protecting other tissues from triglyceride accumulation and insulin resistance [51,52]. Experimental studies have revealed that miR-10b induces steatosis by directly targeting PPAR- $\alpha$ and preventing its expression. PPAR- $\alpha$ was also reported to be specifically regulated by two other miRNAs, miR21 and miR-27b, in the liver [22]. Expression of precursors or antisense nucleotides for miR-21, or miR-27b, in Huh-7 hepatoma cells could significantly modulate the expression of PPAR- $\alpha$ protein, but not its mRNA, suggesting a blockade of PPAR mRNA translation by miR-21/-27b [53]. There are many miRNAs that have been experimentally demonstrated to be able to affect the expression of PPARs in pathological situations. The results from recent studies on the interaction of miRNAs and PPARs are summarized in Table 2.

\section{miRNA signatures for the transition from hepatic steatosis to steatohepatitis}

$\mathrm{NASH}$, is a specific stage between steatosis and cirrhosis in the NAFLD development, characterized by steatosis, lobular inflammation, hepatocellular ballooning and fibrosis histologically [54]. NASH consists of a third of NAFLD cases. As the risk for cirrhosis and hepatocellular carcinoma is of great concern in patients suffering from NASH, prediction of the transition from NAFLD to NASH is of clinical importance. Conventional liver tests usually do not discriminate between steatosis and NASH. Recently there is a growing body of evidence supporting a significant role of miRNAs in the NAFLD pathogenesis and the progression to NASH, and suggesting their use as targets, biomarkers and potential therapeutic tools. Investigation on a specific hepatic miRNA signature (miR-219-5p and miR-199a-5p) demonstrated that they reduce inflammatory adipokine mRNA and protein expression and macrophage innate immune response [55]. In addition, miR-208b-3p, miR-490, miR-185*, miR-199a-5p and miR-214 were consis- 


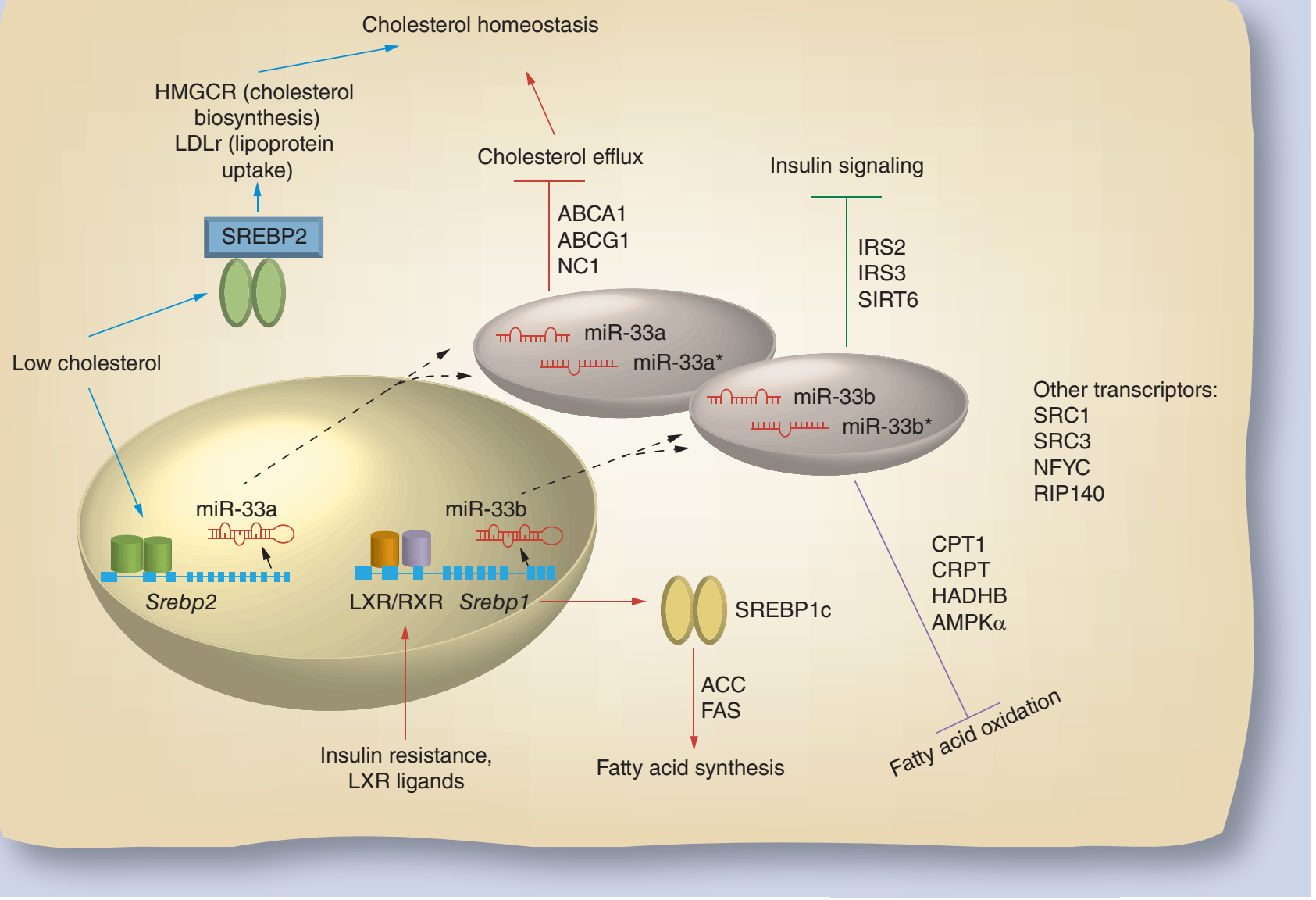

Figure 2. Cross-talk between miR-33 and their target genes in the mechanistic pathway of hepatosteatosis. hsa-miR-33a and hsa-miR-33b, intronic microRNAs (miRNAs) located within the sterol regulatory element-binding protein 2 and 1 genes (SREBP-2 and -1). The sterol-regulatory element-binding protein (SREBP) transcription factors act coordinately with their intronic miRNAs to regulate cholesterol homeostasis, insulin signaling pathway. Both miR-33a and miR-33b duplex arms (their sister strands miR-33a* and $\mathrm{miR}-33 \mathrm{~b}^{*}$ ) were enriched in lipid and specifically fatty acid. For example miR-33a and its sister arm miR-33a* regulate cholesterol mobilization through interaction with genes Abca1, ABCG1 and Npc1. While miR-33b and miR-33b* regulate fatty acid metabolism through regulation of genes ACC, FAS and insulin signaling Irs3, SIRT6. In addition, several transcriptional regulators of lipid metabolism genes ( $\mathrm{SrC1}$, SrC3, Rip140 and $\mathrm{Nfyc}$ ) were also predicted to be targets of both passenger strands.

tently deregulated in serum and liver of steatohepatitis rat, suggesting their potential importance in disease progression [56]. DiSefano et al. identified a panel of miRNAs dysregulated related to liver fibrosis in NAFLD patients with liver fibrosis. In comparison with patients without liver fibrosis, there were nine miRNAs differentially expressed in fibrotic liver. Among of these nine, miR-182, miR-31, miR-183, miR-224 and miR-150 were upregulated, while miR590, miR-378i, miR-17 and miR-219a were downregulated [57]. Moreover, miR-122, the most extensively studied liver specific miRNA, was reported to be significantly decreased in steatohepatitis in the fibrotic liver of mouse fed with methionine-choline-deficient after eight week [58] and demonstrated correlation with the grade of severity of the steatosis [59].

\section{MiRNAs in the progress of liver fibrosis}

Long-term liver damage will progress to liver fibrosis with accumulation of extracellular matrix ECM protein. Activated hepatic stellate cells (HSCs), portal fibroblasts and myofibroblasts of bone marrow origin have been identified as major collagen-producing cells in the injured liver. As many fibrogenic pathways are conserved across tissues, recent findings in the kidney and cardiac system could be extended to studies of fibrosis in the liver. HSCs are activated by fibrogenic cytokines such as TGF- $\beta 1$, angiotensin II and leptin [60]. The secretion of TGF- $\beta$ in particular leads to transdifferentiation of HSCs to promote myofibroblast activation [61]. TGF- $\beta$ regulates the expression of numerous miRNAs - inducing some and suppressing others - such that TGF- $\beta$ is one of the most extensively 


\begin{tabular}{|c|c|c|c|}
\hline Subuint of PPAR & miRNA & Interaction & Ref. (PMID) \\
\hline PPAR $\gamma$ & miR-540 & Suppression & 25560764 \\
\hline PPAR $\alpha$ & miR-199 & Activation & 25389292 \\
\hline PPAR $\gamma$ & miR-139-5p & Suppression & 25536154 \\
\hline PPAR $\gamma$ & miR-130b & Suppression & 25387077 \\
\hline PPAR $\gamma$ & $188-3 p$ & Suppression & 25378159 \\
\hline PPAR $\gamma$ & miR-302a & Suppression & 25109777 \\
\hline PPAR $\alpha$ & miR-143 & Activation & 25012546 \\
\hline PPAR $\gamma$ & $\operatorname{miR}-548 d-5 p$ & Suppression & 24929254 \\
\hline PPAR $\gamma$ & miR-613 & Suppression & 24751522 \\
\hline PPAR $\gamma$ & $\operatorname{miR}-378$ & Suppression & 24666251 \\
\hline PPAR $\alpha$ & miR-518d & Suppression & 24639097 \\
\hline PPAR $\gamma$ & miR-33b & Suppression & 24398549 \\
\hline PPAR $\gamma$ & miR-130b & Suppression & 24311275 \\
\hline PPAR $\alpha$ & miR-33b & Suppression & 24100264 \\
\hline PPAR $\gamma$ & miR-199a & Suppression & 24011070 \\
\hline PPAR $\alpha$ & $\operatorname{miR}-27$ & Suppression & 23897856 \\
\hline PPAR $\gamma$ & miR-122 & Enhanced & 23703729 \\
\hline PPAR $\gamma$ & miR-9 & Suppression & 23525285 \\
\hline PPAR $\gamma$ & miR-374 & Upregualtion & 23468252 \\
\hline PPAR $\alpha$ & miR-21 & Suppression & 20956972 \\
\hline PPAR $\alpha$ & miR-10b & Activation & 19780876 \\
\hline PPAR $\alpha$ & miR-335 & Activation & 22706233 \\
\hline PPAR $\gamma$ & $\operatorname{miR}-132$ & Suppression & 23818645 \\
\hline
\end{tabular}

investigated pathways in miRNA biology [62]. Other miRNA species that are also highly expressed in the healthy liver, for example, miR-125b or miR-22, were shown to be additionally reduced in fibrotic liver biopsies. Notably, accumulating studies have demonstrated that the miR-29 family critically participates in the development of liver fibrosis, renal fibrosis, pulmonary fibrosis and cardiac fibrosis [63]. The miR-29 family is composed of miR-29a, miR-29b and miR-29c, encoded and transcribed in tandem by two genes located on chromosome7 or chromosome1, respectively [64]. miR-29 acted as an antifibrogenic mediator by interfering with profibrogenic cell communication via PDGF-C and IGF-I [64]. Taken together, miR-29 acts as antifibrogenic miRNA by two pathways: by inhibition of ECM formation and interfering with the profibrogenic cell communication pathways via PDGF-B and PDGF-C signaling. In line with these early observations revealed a pivotal role of miR-29 family in negatively regulating HSC activation, further functional analysis to determine the precise roles of miR-29 family in liver fibrosis could provide a novel therapeutic strategy for treating liver fibrosis. Figure 3 illustrates the cross-talk between members of the canonical TGF- $\beta$ signaling and the miRNA machinery. Previous studies have showed that during HSC activation in primary culture, miR-29b overexpression markedly attenuated Colla1 and Colla2 mRNAs expression and additionally inhibited the increased expression of a-SMA, DDR2, FN1, ITGB1 and $P D G F R-\beta$, which were the key genes involved in the activation of HSCs.

Taken together, miRNAs contribute almost every step of the progression of NAFLD. In addition, they are receiving growing attention as to identify novel therapeutic targets of NAFLD. Because no proven pharmacological drug ameliorates NAFLD, it is imperative to design new therapeutic drugs that target key factors of its pathogenesis to ameliorate or prevent disease progression.

\section{miRNA in drug-induced liver steatosis}

Drug-induced liver steatosis (DIS) is a group of heterogeneous disorders that are characterized by fat 
accumulation in hepatocytes and with many features common to NAFLD. Currently, there are no reliable biomarkers to predict the course, severity or prognosis of DIS. miRNAs and their application in DILI have been comprehensively reviewed [65]. PPARs regulation was found to be associated with DILI. For instance, troglitazone, the first PPAR- $\gamma$ agonist that was approved by the US FDA for the treatment of Type 2 diabetes, was withdrawn shortly from the market after receiving several reports of severe liver failure causing death [66]. Liver-specific miRNAs, such as miR-122, miR-21 and miR-192, are believed to repress a set of target mRNAs and thereby regulate specific cellular proteins and cell phenotypes [67,68]. Expression pro- files of such miRNAs are currently being established to identify noninvasive biomarkers for human diseases [69]. There has been interesting growth in the potential of miRNAs as biomarkers of DILI due to detection of a number of miRNAs in various body fluids [70-72]. Several platforms have been developed and successfully applied for quantification of miRNA expression. However, quantifiable performance metrics for these platforms are often ill-defined or simply nonexistent, which hampers informed selection of the most appropriate method, considering the particular study objectives. In light of the growing understanding of miRNAs associated with the pathophysiological mechanisms of liver disease and the bringing of these

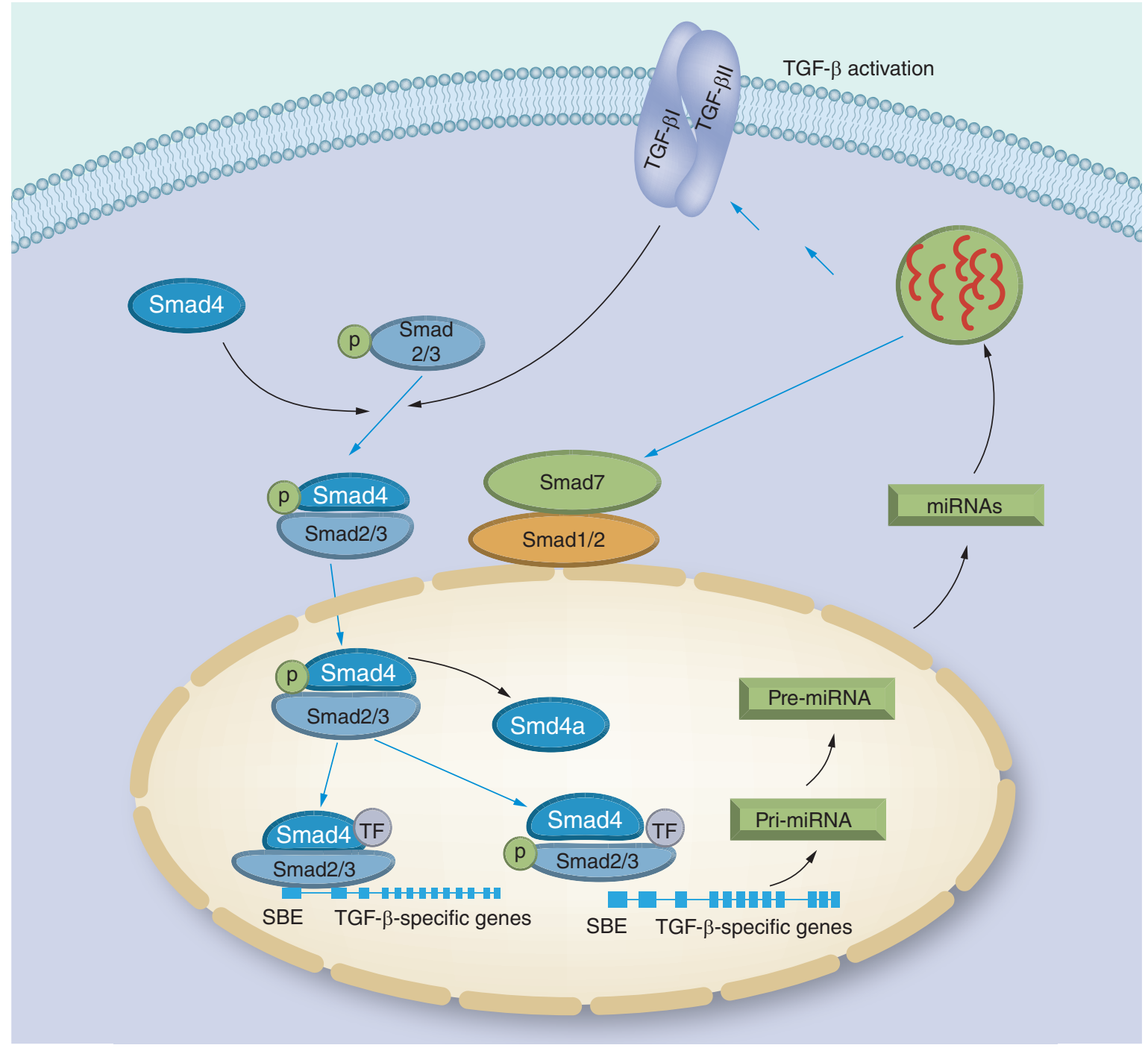

Figure 3. Outline of the crosstalk between members of the canonical TGF- $\beta$ signaling and the miRNA machinery. Transcriptional regulation of miRNAs by Smads largely resembles the canonical TGF- $\beta$ signaling pathway. Signal transduction leads to phosphorylation of an R-Smad, which induces the formation of an R-Smad/co-Smad heterodimer. This complex is imported into the nucleus, binds the SBE and regulates. Phosphorylation of an R-Smad induces nuclear localization. In the nucleus, R-Smad recognizes and binds an SBE-like sequence localized in the stem region of the pri-miRNA, and recruits the Drosha/DGCR8 microprocessor complex to the pri-miRNA. 
Table 3. Circulating miRNAs detected from serum in nonalcoholic fatty liver disease patients.

\begin{tabular}{|c|c|c|c|c|}
\hline miRNAs & Direction & Source & Phenotype & PMID no. \\
\hline \multirow[t]{7}{*}{ miR-122 } & Up & Serum & NAFLD patient & 25386083 \\
\hline & Up & Serum & NAFLD patient & 24973316 \\
\hline & Up & Serum & NAFLD patient & 24217942 \\
\hline & Up & Serum & NAFLD patient & 23727030 \\
\hline & Up & Serum & NAFLD patient & 21886843 \\
\hline & Up & Serum & NAFLD patient & 21886843 \\
\hline & Up & Serum & NAFLD patient & 25141008 \\
\hline miR-103 & Up & Serum & NAFLD patient & 25593466 \\
\hline $\operatorname{miR}-125-5 p$ & Up & Serum & NAFLD patient & 24973316 \\
\hline miR-1290 & Up & Serum & NAFLD patient & 25141008 \\
\hline miR-146b & Up & Serum & NAFLD patient & 25232454 \\
\hline miR-16 & Up & Serum & NAFLD patient & 21886843 \\
\hline miR-181d & Up & Serum & NAFLD patient & 25232454 \\
\hline \multirow[t]{2}{*}{ miR-192-5p } & Up & Serum & NAFLD patient & 25141008 \\
\hline & Up & Serum & NAFLD patient & 24973316 \\
\hline miR-197 & Up & Serum & NAFLD patient & 25232454 \\
\hline miR-19a & Up & Serum & NAFLD patient & 24973316 \\
\hline \multirow[t]{2}{*}{ miR-21 } & Up & Serum & NAFLD patient & 23727030 \\
\hline & Up & Serum & NAFLD patient & 21886843 \\
\hline miR-224 & Up & Serum & NAFLD patient & 25386083 \\
\hline miR-27b-3p & Up & Serum & NAFLD patient & 25141008 \\
\hline miR-33a & Up & Serum & NAFLD patient & 25386083 \\
\hline \multirow[t]{3}{*}{ miR34a } & Up & Serum & NAFLD patient & 23727030 \\
\hline & Up & Serum & NAFLD patient & 21886843 \\
\hline & Up & Serum & NAFLD patient & 21886843 \\
\hline miR-375 & Up & Serum & NAFLD patient & 24973316 \\
\hline miR-451 & Up & Serum & NAFLD patient & 23727030 \\
\hline miR-99a & Down & Serum & NAFLD patient & 25232454 \\
\hline miR-15b & Up & Serum & NAFLD patient & 11994399 \\
\hline
\end{tabular}

miRNAs into clinical use as translational biomarker for DILI, cross-species validation, screening of additional hepatotoxicants and mechanistic-based information of corresponding miRNAs to DILI need to be investigated in the future.

Future perspective: pitfalls \& limitation in translation from potential miRNAs biomarker discovery to clinical utility in predictive \& personalized medicine

Search of the scientific literature results in thousands of papers in the area of biomarker discovery, but only few clinical useful biomarkers have been successful qualified and translated into routine clini- cal practice. Most reported miRNA biomarkers are inadequate to replace the existing clinical test or they are only useful for detecting advanced disease stage. MiRNAs play critical roles throughout cellular development and functional regulations at multiple stages of biological development and disease progress. MiRNA profiling has demonstrated its capacity for subclassifying tumors and monitoring of therapeutic effects. Different expression profiles of miRNAs in diseases and the stability of circulating miRNAs potentially provide a clinically accessible molecular monitoring tool of disease tissues and its response to therapies. Recent publications have reported that miRNAs can enter the circulation system including 
blood and other body fluids. This has been supported by many other studies demontsrating that cells can actively secrete miRNAs into the circulation system via exosomes, which are 30-90 $\mathrm{nm}$ vesicles secreted by a wide range of mammalian cell types and release contents outside of the cell after multivesicular bodies from endosomes fuse with the plasma membrane. A list of circulating miRNAs which have been detected from patients diagnosed with NAFLD are presented in Table 3. Although the detailed mechanism of miRNA secretion needs to be further elucidated, secretory miRNAs are likely a new class of biomessengers. Several miRNA biomarkers that are associated with inflammation, ECM and apoptosis have been studied for their performances in distinguishing NASH from simple steatosis. However, the challenge of circular miRNAs as biomarkers for clinical application remains. These challenges come from almost every step of miRNAs measurement, from pre-ana- lytical, analytical to reproducibility and translation of findings into the real clinical world.

\section{Disclaimer}

The views presented in this article do not necessarily reflect current or future opinion or policy of the US Food and Drug Administration. Any mention of commercial products is for clarification and not intended as endorsement.

\section{Financial \& competing interests disclosure}

The authors have no relevant affiliations or financial involvement with any organization or entity with a financial interest in or financial conflict with the subject matter or materials discussed in the manuscript. This includes employment, consultancies, honoraria, stock ownership or options, expert testimony, grants or patents received or pending, or royalties.

No writing assistance was utilized in the production of this manuscript.

\section{Executive summary}

\section{Background}

- Nonalcoholic fatty liver disease (NAFLD) represents a major concern in the field of liver disease and a public health challenge around the world; it is pandemic and affects both developed and developing countries.

- Many factors have been reported to be associated with the development of NAFLD, including genetic predisposition, life style and environmental exposures.

- Pathogenesis of this disease remains unclear.

miRNAs play an important role in each step of the progression of NAFLD

- Emerging evidence has implicated a role for miRNAs that regulate gene expression either transcriptionally, through targeting of promoter regions, or post-transcriptionally, by blocking translation or promoting cleavage of specific target mRNAs.

- Extensive research efforts are ongoing to identify biological targets for elucidating the mechanisms of progression from the early stage of liver steatosis to more advanced inflammation and fibrosis.

- There is great promise of miRNAs as potential biomarkers for the diagnosis and prognosis of NAFLD.

miRNAs in drug-induced steatosis

- Drug-induced steatosis is a group of heterogeneous disorders and shares many similar features common to NAFLD.

- There is growing interest in the potential of miRNAs as biomarkers of drug-induced liver injury due to a number of miRNAs which have been detected in various body fluids.

- However, cross-species validation, screening of additional hepatotoxicants and mechanistic-based information of corresponding miRNAs to drug-induced liver injury needs to be investigated in the future in order to bring these potential biomarkers into the real clinical setting.

\section{Future perspective}

- MiRNAs play critical roles throughout cellular development and functional regulations at multiple stages of biological development and disease progress.

- MiRNA profiling has demonstrated its capacity for subclassifying tumors and monitoring of therapeutic effects.

- We anticipate that such knowledge will facilitate the development of improved methods for diagnosis and clinical monitoring and eventually be translated into the development of novel treatment strategies for this increasingly important disease.

\section{References}

Papers of special note have been highlighted as: • of interest;

•• of considerable interest

1 Ahmed M. Non-alcoholic fatty liver disease in 2015. World J. Hepatol. 7(11), 1450-1459 (2015).
2 Sun C, Fan JG, Qiao L. Potential epigenetic mechanism in non-alcoholic fatty liver disease. Int. J. Mol. Sci. 16(3), 5161-5179 (2015).

3 Carthew RW, Sontheimer EJ. Origins and mechanisms of miRNAs and siRNAs. Cell 136(4), 642-655 (2009). 
4 Londin E, Loher P, Telonis AG et al. Analysis of 13 cell types reveals evidence for the expression of numerous novel primate- and tissue-specific microRNAs. 112(10), E1106-E1115 (2015).

- This study indicated that there are significant number of tissue-specific miRNAS are uncharacterized.

5 Ameres SL, Zamore PD. Diversifying microRNA sequence and function. Nat. Rev. Mol. Cell Biol. 14(8), 475-488 (2013).

6 Hausser J, Zavolan M. Identification and consequences of miRNA-target interactions - beyond repression of gene expression. Nat. Rev. Genet. 15(9), 599-612 (2014).

7 Kong YW, Ferland-Mccollough D, Jackson TJ, Bushell M. MicroRNAs in cancer management. Lancet Oncol. 13(6), e249-258 (2012).

8 Kamal MA, Mushtaq G, Greig NH. Current update on synopsis of miRNA dysregulation in neurological disorders. CNS Neurol. Dis. Drug Target. 14(4), 492-501 (2015).

9 Oliveira-Carvalho V, Da Silva MM, Guimaraes GV, Bacal F, Bocchi EA. MicroRNAs: new players in heart failure. Mol. Biol. Rep. 40 (3), 2663-2670 (2013).

10 Lamontagne J, Steel LF, Bouchard MJ. Hepatitis B virus and microRNAs: complex interactions affecting hepatitis B virus replication and hepatitis B virus-associated diseases. World J. Gastroenterol. 21(24), 7375-7399 (2015).

11 Baldeon RL, Weigelt K, De Wit $\mathrm{H}$ et al. Type 2 diabetes monocyte microRNA and mRNA expression: dyslipidemia associates with increased differentiation-related genes but not inflammatory activation. PLoS ONE 10(6), e0129421 (2015).

12 Zhou B, Li Z, Yang H, He N. Extracellular miRNAs: origin, function and biomarkers in hepatic diseases. J. Biomed. Nanotechnol. 10 (10), 2865-2890 (2014).

13 Krauskopf J, Caiment F, Claessen SM et al. Application of high-throughput sequencing to circulating microRNAs reveals novel biomarkers for drug-induced liver injury. Toxicol. Sci. 143(2), 268-276 (2015).

14 Ayyadurai S, Charania MA, Xiao B, Viennois E, Zhang Y, Merlin D. Colonic miRNA expression/secretion, regulated by intestinal epithelial pept1, plays an important role in cell-to-cell communication during colitis. PLoS ONE 9(2), e87614 (2014).

15 Kogure T, Costinean S, Yan I, Braconi C, Croce C, Patel T. Hepatic miR-29AB1 expression modulates chronic hepatic injury. J. Cell. Mol. Med. 16(11), 2647-2654 (2012).

16 Scherr M, Elder A, Battmer K, Barzan D, Bomken S. Differential expression of miR-17-92 identifies BCL2 as a therapeutic target in BCR-ABL-positive B-lineage acute lymphoblastic leukemia. 28(3), 554-565 (2014).

17 Bostjancic E, Glavac D. Mirnome in myocardial infarction: future directions and perspective. World. J. Cardiol. 6(9), 939-958 (2014).

18 Chen L, Hou J, Ye L et al. MicroRNA-143 regulates adipogenesis by modulating the MAP2K5-ERK5 signaling. Sci. Rep. 4, 3819 (2014).

19 Christian P, Su Q. MicroRNA regulation of mitochondrial and er stress signaling pathways: implications for lipoprotein metabolism in metabolic syndrome. Am. J. Physiol. Endocrinol. Metab. 307(9), E729-737 (2014).

20 Mattis AN, Song G, Hitchner K et al. A screen in mice uncovers repression of lipoprotein lipase by microRNA-29a as a mechanism for lipid distribution away from the liver. Hepatol. (Baltimore, MD) 61(1), 141-152 (2015).

21 Chakraborty C, Doss CG, Bandyopadhyay S, Agoramoorthy G. Influence of miRNA in insulin signaling pathway and insulin resistance: micro-molecules with a major role in Type-2 diabetes. Wiley Interdiscip. Rev. RNA 5(5), 697-712 (2014).

22 Panera N, Gnani D, Crudele A, Ceccarelli S, Nobili V, Alisi A. MicroRNAs as controlled systems and controllers in nonalcoholic fatty liver disease. World J. Gastroenterol. 20(41), 15079-15086 (2014).

23 Itoh M, Kato H, Suganami T et al. Hepatic crown-like structure: a unique histological feature in non-alcoholic steatohepatitis in mice and humans. PLoS ONE 8(12), e82163 (2013).

24 Li Y, Kowdley KV. MicroRNAs in common human diseases. Genom. Proteom. Bioinform. 10(5), 246-253 (2012).

25 Sanyal AJ, Brunt EM, Kleiner DE et al. Endpoints and clinical trial design for nonalcoholic steatohepatitis. Hepatol. (Baltimore, MD) 54(1), 344-353 (2011).

26 White DL, Kanwal F, El-Serag HB. Association between nonalcoholic fatty liver disease and risk for hepatocellular cancer, based on systematic review. Clin. Gastroenterol. Hepatol. 10 (12), 1342-1359.e1342 (2012).

27 Gerhard GS, Distefano JK. Micro RNAs in the development of non-alcoholic fatty liver disease. World J. Hepatol. 7(2), 226-234 (2015).

-. Summarizes the current founding of miRNAs involved in the steatosis classified by in vitro and in vivo models.

28 Chen Y, Verfaillie CM. MicroRNAs: the fine modulators of liver development and function. Liver Int. 34(7), 976-990 (2014).

29 Price NL, Ramirez CM, Fernandez-Hernando C. Relevance of microRNA in metabolic diseases. Crit. Rev. Clin. Lab. Sci. 51(6), 305-320 (2014).

30 Williams JA, Manley S, Ding WX. New advances in molecular mechanisms and emerging therapeutic targets in alcoholic liver diseases. World J. Gastroenterol. 20 (36), 12908-12933 (2014).

31 Singaravelu R, Chen R, Lyn RK et al. Hepatitis C virus induced up-regulation of microRNA-27: a novel mechanism for hepatic steatosis. Hepatol. (Baltimore, MD) 59(1), 98-108 (2014).

32 Vickers KC, Sethupathy P, Baran-Gale J, Remaley AT. Complexity of microRNA function and the role of isomirs in lipid homeostasis. J. Lipid Res. 54(5), 1182-1191 (2013).

33 Li T, Francl JM, Boehme S, Chiang JY. Regulation of cholesterol and bile acid homeostasis by the cholesterol 7alpha-hydroxylase/steroid response element-binding protein 2/microRNA-33a axis in mice. Hepatol. (Baltimore, $M D$ ) 58(3), 1111-1121 (2013).

34 Horie T, Nishino T, Baba O et al. MicroRNA-33 regulates sterol regulatory element-binding protein 1 expression in mice. Nat. Comm. 4, 2883 (2013). 
-. The results of this study elucidated a novel interaction between SREBP-1 and SREBP-2 mediated by miR-33 in vivo.

Goedeke L, Vales-Lara FM, Fenstermaker $\mathrm{M}$ et al. A regulatory role for microRNA $33^{*}$ in controlling lipid metabolism gene expression. Mol. Cell. Biol. 33(11), 2339-2352 (2013). metabolic syndrome: a systematic review. ARYA Atheroscler. 9(6), 372-376 (2013).

37 Ramirez CM, Goedeke L, Rotllan N et al. MicroRNA 33 regulates glucose metabolism. Mol. Cell. Biol. 33(15), 2891-2902 (2013).

38 Cirera-Salinas D, Pauta M, Allen RM et al. Mir-33 regulates cell proliferation and cell cycle progression. Cell Cycle 11(5), 922-933 (2012).

39 Lodhi IJ, Semenkovich CF. Peroxisomes: a nexus for lipid metabolism and cellular signaling. Cell Metab. 19(3), 380-392 (2014).

40 Grygiel-Gorniak B. Peroxisome proliferator-activated receptors and their ligands: nutritional and clinical implications - a review. Nutr. J. 13, 17 (2014).

41 Song K, Han C, Zhang J et al. Epigenetic regulation of microRNA-122 by peroxisome proliferator activated receptorgamma and hepatitis B virus $\mathrm{x}$ protein in hepatocellular carcinoma cells. Hepatology 58(5), 1681-1692 (2013).

42 Peyrou M, Ramadori P, Bourgoin L, Foti M. Ppars in liver diseases and cancer: epigenetic regulation by microRNAs. PPAR Res. 2012757803 (2012).

43 Escalona-Nandez I, Guerrero-Escalera D, EstanesHernandez A, Ortiz-Ortega V, Tovar AR, Perez-Monter C. The activation of peroxisome proliferator-activated receptor gamma is regulated by Kruppel-like transcription factors 6 \& 9 under steatotic conditions. Biochem. Biophys. Res. Comm. 458(4), 751-756 (2015).

44 Giby VG, Ajith TA. Role of adipokines and peroxisome proliferator-activated receptors in nonalcoholic fatty liver disease. World. J. Hepatol. 6(8), 570-579 (2014).

45 Lima-Cabello E, Garcia-Mediavilla M, Miquilena-Colina M et al. Enhanced expression of pro-inflammatory mediators and liver x-receptor-regulated lipogenic genes in nonalcoholic fatty liver disease and hepatitis c. Clin. Sci. 120, 239-250 (2011).

46 Yang T, Li X, Zhu W et al. Alteration of antioxidant enzymes and associated genes induced by grape seed extracts in the primary muscle cells of goats in vitro. PLoS ONE 9(9), e107670 (2014).

47 Lehmann M, Pirinen E, Mirsaidi A et al. ARTD1-induced poly-ADP-ribose formation enhances ppargamma ligand binding and co-factor exchange. Nucl. Acids Res. 43(1), 129-142 (2015).

48 Gao Y, Shen W, Lu B, Zhang Q, Hu Y, Chen Y. Upregulation of hepatic VLDLR via PPARalpha is required for the triglyceride-lowering effect of fenofibrate. J. Lipid Res. 55(8), 1622-1633 (2014).

49 Roberts LD, Bostrom P, O'Sullivan JF et al. Betaaminoisobutyric acid induces browning of white fat and hepatic beta-oxidation and is inversely correlated with cardiometabolic risk factors. Cell Metab. 19(1), 96-108 (2014).

50 Pawlak M, Lefebvre P, Staels B. Molecular mechanism of pparalpha action and its impact on lipid metabolism, inflammation and fibrosis in non-alcoholic fatty liver disease. J. Hepatol. 62(3), 720-733 (2015).

51 Kurtz M, Capobianco E, Careaga V et al. Peroxisome proliferator-activated receptor ligands regulate lipid content, metabolism, and composition in fetal lungs of diabetic rats. J. Endocrinol. 220(3), 345-359 (2014).

52 Albert JS, Yerges-Armstrong LM, Horenstein RB et al. Null mutation in hormone-sensitive lipase gene and risk of type 2 diabetes. N. Engl. J. Med. 370 (24), 2307-2315 (2014).

53 Zhou J, Wang KC, Wu W et al. MicroRNA-21 targets peroxisome proliferators-activated receptor-alpha in an autoregulatory loop to modulate flow-induced endothelial inflammation. Proc. Natl Acad. Sci. USA 108(25), 1035510360 (2011).

54 Takahashi Y, Fukusato T. Histopathology of nonalcoholic fatty liver disease/nonalcoholic steatohepatitis. World J. Gastroenterol. 20(42), 15539-15548 (2014).

55 Rius B, Titos E, Moran-Salvador E et al. Resolvin d1 primes the resolution process initiated by calorie restriction in obesity-induced steatohepatitis. FASEB J. 28(2), 836-848 (2014).

56 Chen YP, Jin X, Xiang Z, Chen SH, Li YM. Circulating microRNAs as potential biomarkers for alcoholic steatohepatitis. Liver Int. 33(8), 1257-1265 (2013).

57 Leti F, Malenica I, Doshi M et al. High-throughput sequencing reveals altered expression of hepatic microRNAs in nonalcoholic fatty liver disease-related fibrosis. Translat. Res. (2015).

58 Csak T, Bala S, Lippai D et al. MicroRNA-122 regulates hypoxia-inducible factor-1 and vimentin in hepatocytes and correlates with fibrosis in diet-induced steatohepatitis. Liver Int. 35(2), 532-541 (2015).

59 Enache LS, Enache EL, Ramiere C et al. Circulating RNA molecules as biomarkers in liver disease. Int. J. Mol. Sci. 15(10), 17644-17666 (2014).

60 Elpek GO. Cellular and molecular mechanisms in the pathogenesis of liver fibrosis: an update. World J. Gastroenterol. 20(23), 7260-7276 (2014).

61 Pellicoro A, Ramachandran P, Iredale JP, Fallowfield JA. Liver fibrosis and repair: immune regulation of wound healing in a solid organ. Nat. Rev. Immunol. 14(3), 181-194 (2014).

62 Lee SH, Do SI, Kim HS. Hyperoxia accelerates progression of hepatic fibrosis by up-regulation of transforming growth factor-beta expression. World J. Gastroenterol. 20(11), 3011-3017 (2014).

63 Tan J, Tong BD, Wu YJ, Xiong W. MicroRNA-29 mediates TGFbeta1-induced extracellular matrix synthesis by targeting wnt/beta-catenin pathway in human orbital fibroblasts. Int. J. Clin. Exp. Pathol. 7(11), 7571-7577 (2014).

64 Kwiecinski M, Elfimova N, Noetel A et al. Expression of platelet-derived growth factor- $\mathrm{C}$ and insulin-like growth 
factor I in hepatic stellate cells is inhibited by miR-29. Lab Invest. 92(7), 978-987 (2012).

65 Wang Y, Chen T, Tong W. MiRNAs and their application in drug-induced liver injury. Biomark. Med. 8(2), 161-172 (2014).

66 Jaeschke H. Troglitazone hepatotoxicity: are we getting closer to understanding idiosyncratic liver injury? Toxicol. Sci. 97(1), 1-3 (2007).

67 Alizadeh E, Akbarzadeh A, Eslaminejad MB et al. Up regulation of liver-enriched transcription factors HNF4a and HNF6 and liver-specific microRNA (miR-122) by inhibition of Let-7b in mesenchymal stem cells. Chem. Biol. Drug Design 85(3), 268-279 (2015).

68 Klaassen CD, Lu H, Cui JY. Epigenetic regulation of drug processing genes. Toxicol. Mech. Method. 21(4), 312-324 (2011).
69 Sawant DV, Yao W, Wright Z et al. Serum microRNA-21 as a biomarker for allergic inflammatory disease in children. MicroRNA 4(1), 36-40 (2015).

70 Wu K, Li L, Li S. Circulating microRNA-21 as a biomarker for the detection of various carcinomas: an updated metaanalysis based on 36 studies. Tumour Biol. 36(3), 1973-1981 (2015).

71 Witwer KW. Circulating microRNA biomarker studies: pitfalls and potential solutions. Clin. Chem. 61(1), 56-63 (2015).

72 Tang G, Shen X, Lv K, Wu Y, Bi J, Shen Q. Different normalization strategies might cause inconsistent variation in circulating microRNAs in patients with hepatocellular carcinoma. Med. Sci. Monitor. 21, 617-624 (2015). 